\title{
Multisensor ESA Resource Management
}

\author{
Gregory A. Watson \\ Code B32 \\ Systems Research and Technology Department \\ Naval Surface Warfare Center Dahlgren Division \\ Dahlgren, Virginia 22448-5100 \\ 540-653-7378, gawatso@nswc.navy.mil
}

\begin{abstract}
The integration of multiple sensors with an Electronically Scanned Array (ESA) for target tracking and resource management has been intensely investigated, and several effective techniques have been developed. Conventional techniques employ complex, decision-directed logic to select the target revisit interval and adjust the filter process noise to account for target maneuvers, but have the potential to improve performance. For most systems, each sensor provides its information to a central location where the integration occurs. This central track is employed for system decisions and provides a manageable tracking environment but restricts the potential for system improvement. An ESA is highly controllable and has the ability to greatly enhance tracking performance. Resource allocation for an ESA is critical, since it must support multiple functions, and several modern techniques have been developed to enhance its performance as a stand-alone sensor by effectively managing its time-energy budget. The integration of an ESA with other sensors can further enhance the tracking and reduce the resource allocation requirements of the ESA. This paper presents a technique for ESA resource management through the use of multisensor integration that employs the Interacting Multiple Model (IMM) algorithm and the Probabilistic Data Association Filter (PDAF). Simulation results are provided to demonstrate the effectiveness of this modern integration technique.
\end{abstract}

\section{TABLE OF CONTENTS}

\section{Introduction}

2. Background

3. Probabilistic Data Association Filter

4. Multisensor Integration of ESA Track

5. Interacting Multiple Model Algorithm

6. ESA Revisit Technique

7. Simulation Results

8. Summary and Concluding Remarks

This work was supported by the AEGIS Program Office at the Naval Surface Warfare Center Dahlgren Division, Dahlgren, Virginia.

\section{INTRODUCTION}

The integration of multiple sensors for target tracking has been intensely investigated in recent years and several techniques have been developed to improve the performance against maneuvering targets. Situational awareness has also become important due to the increasing complexity of the battle space. Through effective sensor integration, resource management and situational awareness can also be enhanced. For most techniques, a central track is updated with the information (i.e., measurements, estimates, etc.) provided by the sensor suite, and this track is employed for system decisions and is usually not used by the individual sensors. This low level of sensor integration provides a safe and manageable tracking environment but restricts the potential for system improvement by not exploiting the potential synergism among the sensors. These techniques are also very complex, since they use decision-directed logic to select the target revisit interval and adjust the process noise of the filter to account for target maneuvers. A single model Kalman filter is typically employed and its process noise must be continually adjusted to account for data rate, measurement accuracy, sensor type, target maneuverability, etc. The accuracy of the state estimates for the central track can be less accurate than the local track, if the process noise is not properly adjusted to account for these factors [1].

The Interacting Multiple Model (IMM) algorithm is an effective technique for tracking targets flying at high speeds and performing maneuvers $[2,3]$. The IMM algorithm uses multiple models that interact through state mixing to track a target through an arbitrary maneuver. Governed by an underlying Markov chain, the state estimates are mixed according to their model probabilities and model switching probabilities. The output estimate is a probabilistic sum of the individual filter estimates and represents the relative performance of each model. The IMM algorithm provides a flexible method for tracking maneuvering targets by eliminating the need for decision-directed logic. The IMM algorithm is considered a nearly-consistent estimator because it is nearly consistent (i.e., except at the time of the first measurement after a maneuver). Thus, the output er- 
ror covariance of the IMM algorithm reflects the actual tracking performance and can be employed for adjusting the tracking parameters, system decisions, and resource management. The integration of multiple sensors for tracking is also readily accomplished with the IMM algorithm [5]. The use of multiple models eliminates the need for the decision-directed logic required to adjust the process noise for conventional techniques. When tracking with multiple sensors, the state estimates provided by the IMM algorithm are more accurate than traditional methods [5].

Several measurements, resulting from false alarms and clutter, may be available to update a track at a given time. The Nearest Neighbor (NN) data association technique is commonly used when there is more than one contact-to-track association. The NN approach uses the contact nearest the predicted measurement to update the track and does not account for the possibility of an incorrect decision. Probabilistic methods are superior when compared to the NN approach because all hypotheses are assigned probabilities of being correct. The Probabilistic Data Association Filter (PDAF) is a recursive algorithm with fixed computational requirements and provides better performance when compared to the NN technique [4]. The PDAF assumes the target exists and can therefore only be used for maintaining existing tracks. However, track observability can be added to the PDAF to allow for the initiation and termination of tracks [4], where an observable (true or live) target is one having nonzero probability of detection and an unobservable (false or dead) target is one having a probability of detection of zero. The observability concept and the Interacting Multiple Model (IMM) algorithm were combined to form the IMMPDAF $[4,6]$. The IMMPDAF usually combines two PDAFs for each kinematic model: one each for the observable and unobservable hypothesis. After initiation has occurred, the filters containing the unobservable hypotheses are typically removed. For this paper, the tracks are assumed to exist. The IMMPDAF is an effective technique for tracking maneuvering targets in a false alarm and clutter environment and has the ability to perform adaptive sampling for ESA resource allocation.

An Electronically Scanned Array (ESA) supports multiple functions, such as surveillance, weapons control, command and decision, volume and horizon search, etc., and management of its resources is critical. Several techniques have been developed to enhance its performance as a stand-alone sensor by effectively managing the timeenergy budget [6-8]. The integration of an ESA with additional sensors has the potential to further enhance the tracking and reduce the resource allocation requirements of the ESA. Conventional resource allocation approaches require decision-directed logic which further increases the complexity of existing algorithms. In a con- ventional approach, the ESA sets its revisit rate for a target based on function requirements. When a supporting sensor updates the track, a new rate must be determined. In the conventional approach, it is particularly problematic when the supporting sensor fails to report a detection because the existing revisit time must be changed to occur at an earlier time which may be unavailable. If the supporting sensor loses track, it must first determine that the track is lost and communicate the information to the ESA, which then has to set a new rate but may also lose track because of the inherent delay.

The IMM algorithm provides a more effective method for ESA resource management when supported by additional sensors. Unlike conventional schemes, a revisit interval is determined after each ESA track update using the output of the IMM algorithm. When additional sensors support the track, a new revisit interval is computed for the ESA after each track update and the ESA is commanded to delay its initial update a specified amount. This process continues to occur until the ESA is required to perform a dwell. If the supporting sensor fails to report, the ESA has already scheduled a time for its next dwell and the track continues to be updated. The synergy between the sensors is exploited to reduce the resource requirements of the ESA by using the output of the IMM algorithm and computing a revisit interval after each track update. While the revisit technique described in this paper has been successfully demonstrated for a surveillance application, it can also be employed for other tactical functions such as weapons control.

The supporting sensors can be both internal and external to the ESA and this is apparent with the ESA functions of search and track. The search function inevitably obtains measurements from targets that are already in track. These measurements can be employed to provide more accurate state estimates and/or reduce the resource requirements required by the track function. This paper presents a technique for ESA resource management through the use of multisensor integration. Simulation results for a surveillance application are presented to demonstrate the resource allocation technique. The sensor suite for the simulation includes an ESA and a sensor providing angle measurements, which could be an InfraRed Search and Track (IRST) and/or a Precision Electronic Support Measure (PESM).

\section{BACKGROUND}

The conventional discrete-time model for target tracking is typically a linear (or linearized) stochastic system and is given by

$$
\begin{aligned}
X_{k} & =F_{k-1} X_{k-1}+G_{k-1} w_{k-1} \\
Z_{k} & =H_{k} X_{k}+v_{k}
\end{aligned}
$$


where $w_{k-1}$ is an $m \times 1$ process noise vector with $w_{k-1} \sim$ $N\left(0, Q_{k-1}\right), v_{k}$ is an $p \times 1$ measurement error vector with $v_{k} \sim N\left(0, R_{k}\right), X_{k}$ is an $n \times 1$ state vector, and $Z_{k}$ is a $p \times 1$ measurement vector. The Kalman filter algorithm is commonly used to estimate the state and error covariance of the system from the measurements. The equations for the Kalman filter are outlined as follows.

Time Update:

$$
\begin{aligned}
X_{k \mid k-1} & =F_{k-1} X_{k-1 \mid k-1} \\
P_{k \mid k-1} & =F_{k-1} P_{k-1 \mid k-1} F_{k-1}^{T}+G_{k-1} Q_{k-1} G_{k-1}^{T}
\end{aligned}
$$

Measurement Update:

$$
\begin{aligned}
X_{k \mid k} & =X_{k \mid k-1}+K_{k}\left[\widetilde{Z}_{k}\right] \\
P_{k \mid k} & =\left[I-K_{k} H_{k}\right] P_{k \mid k-1}
\end{aligned}
$$

with

$$
\begin{aligned}
\widetilde{Z}_{k} & =Z_{k}-H_{k} X_{k \mid k-1}=Z_{k}-Z_{k \mid k-1} \\
K_{k} & =P_{k \mid k-1} H_{k}^{T} S_{k}^{-1} \\
S_{k} & =H_{k} P_{k \mid k-1} H_{k}^{T}+R_{k}
\end{aligned}
$$

where $X_{i \mid j}$ denotes the state estimate for time $i$ given measurements through time $j$, and $P_{i \mid j}$ denotes the corresponding error covariance. An extended Kalman filter is employed because measurements of the target state include range, bearing, and/or elevation and it is assumed that the false alarm rate is very small. The extended Kalman filter readily accommodates track updates with measurements from dissimilar sensors and has been very effective in an electronic warfare environment that includes jamming and range gate pull-off [7].

\section{Probabilistic Data ASSOCIATION Filter}

The PDAF is used when the target-originated measurement can not be determined with a high degree of confidence. As a result, more than one measurement may be used to update the state. The PDAF is considered an "all-neighbors" approach when compared to the Kalman filter since it avoids making a "hard" decision. The equations for the PDAF are outlined as follows, but for a thorough explanation refer to [4]. The expected state estimate and error covariance are computed according to Eqs. (2.3) and (2.4). The state estimate, $X_{k \mid k}$, is given by

$$
\begin{aligned}
X_{k \mid k} & =\sum_{i=0}^{m_{k}} \beta_{k}^{i} X_{k \mid k}^{i} \\
& =X_{k \mid k-1}+K_{k} \widetilde{Z}_{k}^{c}
\end{aligned}
$$

where

$$
\widetilde{Z}_{k}^{c}=\sum_{i=1}^{m_{k}} \beta_{k}^{i} \widetilde{Z}_{k}^{i}
$$

and $m_{k}$ is the number of validated measurements. The associated error covariance $P_{k \mid k}$ is given by

$$
P_{k \mid k}=\beta_{k}^{0} P_{k \mid k-1}+\left(1-\beta_{k}^{0}\right) P_{k \mid k}^{c}+\widetilde{P}_{k}
$$

where

$$
\tilde{P}_{k}=K_{k}\left(\sum_{i=1}^{m_{k}} \beta_{k}^{i} \tilde{Z}_{k}^{i}\left(\widetilde{Z}_{k}^{i}\right)^{T}-\tilde{Z}_{k}^{c}\left(\widetilde{Z}_{k}^{c}\right)^{T}\right)\left(K_{k}\right)^{T}
$$

and

$$
P_{k \mid k}^{c}=\left[I-K_{k} H_{k}\right] P_{k \mid k-1}
$$

The probabilities concerning the origin of the measurements $\beta_{k}^{i}$ are given by

$$
\begin{aligned}
\beta_{k}^{0} & =\frac{1-P_{D} P_{G}}{1-\delta_{k}} \\
\beta_{k}^{i} & =\frac{P_{D} P_{G} \frac{V_{k}}{\widehat{m}_{k}} \Lambda_{k}^{i}}{1-\delta_{k}}
\end{aligned}
$$

where

$$
\delta_{k}= \begin{cases}P_{D} P_{G} & m_{k}=0 \\ P_{D} P_{G}\left(1-\frac{V_{k}}{\widehat{m}_{k}} \sum_{i=1}^{m_{k}} \Lambda_{k}^{i}\right) & m_{k}>0\end{cases}
$$

with target-oriented measurement probability of detection $P_{D}$ and probability of being in the validation region $P_{G}$. For the validation region, the volume $V_{k}$, the expected number of false measurements $\widehat{m}_{k}$, and the probability density function for the $i^{\text {th }}$ measurement $\Lambda_{k}^{i}$, are given by

$$
\begin{aligned}
\Lambda_{k}^{i} & =\frac{\left(1 / P_{G}\right)}{\sqrt{\left|2 \pi S_{k}\right|} \exp \left\{-\frac{1}{2}\left(g_{k}^{i}\right)^{2}\right\}} \\
V_{k} & =\frac{4}{3} \pi \sqrt{\operatorname{det}\left\{g_{k}^{2} S_{k}\right\}} \\
\hat{m}_{k} & = \begin{cases}0 & m_{k}=0 \\
m_{k}-P_{D} P_{G} & m_{k}>0\end{cases}
\end{aligned}
$$

where

$$
g_{k}^{i}=\left(\tilde{Z}_{k}^{i}\right)^{T}\left(S_{k}\right)^{-1} \tilde{Z}_{k}^{i}
$$

\section{MULTISENSOR INTEGRATION FOR ESA}

Consider a system receiving measurements from $n$ sensors. The set of measurements at time $k$ for an extended Kalman filter can be represented as

$$
\left\{Z_{k}^{i}\right\}=\left\{Y_{k}^{i} h_{k}\left(X_{k}\right)+v_{k}^{i}\right\}
$$

where $i=1, \ldots, n$. The $Y_{k}^{i}$ accounts for the fact that individual sensors may be of different dimension. The time update equations for processing $n$ measurements are given by Eqs. (2.3) and (2.4). The measurement update equations are given by

$$
\begin{aligned}
X_{k \mid k}^{i} & =X_{k \mid k}^{i-1}+K_{k}^{i}\left[\widetilde{Z}_{k}^{i}\right] \\
P_{k \mid k}^{i} & =\left[I-K_{k}^{i} Y_{k}^{i} H_{k}\right] P_{k \mid k}^{i-1}
\end{aligned}
$$


where

$$
\begin{aligned}
\widetilde{Z}_{k}^{i} & =Z_{k}^{i}-Y_{k}^{i} h_{k}\left(X_{k \mid k}^{i-1}\right)=Z_{k}^{i}-Z_{k \mid k}^{i-1} \\
K_{k}^{i} & =P_{k \mid k}^{i-1}\left(Y_{k}^{i} H_{k}\right)^{T}\left(S_{k}^{i}\right)^{-1} \\
S_{k}^{i} & =Y_{k}^{i} H_{k} P_{k \mid k}^{i-1}\left(Y_{k}^{i} H_{k}\right)^{T}+R_{k}^{i}
\end{aligned}
$$

with $X_{k \mid k}^{0}=X_{k \mid k-1}$ and $P_{k \mid k}^{0}=P_{k \mid k-1}$ for the first measurement processed. The $H_{k}$ is the gradient of $h_{k}$ with respect to $X_{k}$. Measurement fusion is an alternative to sequential processing but is not employed in this paper [5].

The dynamics of the target are modeled as in Eqs. (2.1) and (2.2) with the range, bearing, and elevation measurements of the ESA, denoted by E, in track mode given by

$$
Z_{k}^{E}=\left[\begin{array}{lll}
R_{k}^{E} & B_{k}^{E} & E_{k}^{E}
\end{array}\right]^{T}=h_{k}^{E}\left(X_{k}\right)+v_{k}^{E}
$$

where $v_{k}^{E} \sim N\left(0, R_{k}^{E}\right)$. The value of $Y_{k}^{E}$ is a $3 \times 3$ identity matrix and $h_{k}^{E}\left(X_{k}\right)$ is given by

$$
h_{k}^{E}\left(X_{k}\right)=\left[\begin{array}{c}
\sqrt{x_{k}^{2}+y_{k}^{2}+z_{k}^{2}} \\
\tan ^{-1}\left(\frac{y_{k}}{x_{k}}\right) \\
\tan ^{-1}\left(\frac{z_{k}}{\sqrt{x_{k}^{2}+y_{k}^{2}}}\right)
\end{array}\right]
$$

There can be many unique sensors supporting the ESA track and may include a PESM, denoted by $\mathrm{P}$, and an IRST, denoted by I. The search function of the ESA can also be considered a supporting sensor and can provide measurements in two forms: 1) range only as determine from standard search processing, denoted by $\mathrm{S}$, or 2) range, bearing, and elevation as computed with monopulse processing in search, denoted by $M$. These measurements are modeled as

$$
\begin{aligned}
Z_{k}^{P} & =\left[\begin{array}{ll}
B_{k}^{P} & E_{k}^{P}
\end{array}\right]^{T}=h_{k}^{P}\left(X_{k}\right)+v_{k}^{P} \\
Z_{k}^{I} & =\left[\begin{array}{ll}
B_{k}^{I} & E_{k}^{I}
\end{array}\right]^{T}=h_{k}^{I}\left(X_{k}\right)+v_{k}^{I} \\
Z_{k}^{S} & =R_{k}^{S}=h_{k}^{S}\left(X_{k}\right)+v_{k}^{S} \\
Z_{k}^{M} & =\left[\begin{array}{lll}
R_{k}^{M} & B_{k}^{M} & E_{k}^{M}
\end{array}\right]^{T}=h_{k}^{M}\left(X_{k}\right)+v_{k}^{M}
\end{aligned}
$$

where $v_{k}^{i} \sim N\left(0, R_{k}^{i}\right)$, with $i$ referring to the supporting sensor. The values of $Y_{k}^{i}$ are

$$
\begin{aligned}
Y_{k}^{M} & =Y_{k}^{E} \\
Y_{k}^{S} & =\left[\begin{array}{lll}
1 & 0 & 0
\end{array}\right] \\
Y_{k}^{P} & =Y_{k}^{I}=\left[\begin{array}{lll}
0 & 1 & 0 \\
0 & 0 & 1
\end{array}\right]
\end{aligned}
$$

with

$$
h_{k}^{i}\left(X_{k}\right)=Y_{k}^{i} h_{k}^{E}\left(X_{k}\right)
$$

\section{IMM ALGORITHM}

An important problem is the state estimation of a linear system with Markovian switching coefficients. In this problem, the system dynamics are represented by multiple models which are hypothesized to be correct. The IMM algorithm is a modern approach to merging the different model hypotheses. Previous investigations in the literature indicate that the IMM algorithm is the superior multiple model technique [2-5]. A linear system with Markovian switching coefficients can be represented as

$$
\begin{aligned}
X_{k} & =F_{k-1}\left(\theta_{k}\right) X_{k-1}+G_{k-1}\left(\theta_{k}\right) w_{k-1} \\
Z_{k} & =H_{k}\left(\theta_{k}\right) X_{k}+v_{k}
\end{aligned}
$$

where $\theta_{k}$ is a finite state Markov chain taking values in $\{1, \ldots, N\}$ according to the probability, $p_{i j}$, of transitioning from model $i$ to model $j$.

The IMM algorithm consists of a filter for each model, a model probability evaluator, an estimate mixer at the input of the filters, and an estimate combiner at the output of the filters. The multiple models interact through the mixing to track a target maneuvering through an arbitrary trajectory. With the assumption that the switching is governed by an underlying Markov chain, the mixer uses the model probabilities and the model switching probabilities to compute a mixed estimate for each model. At the beginning of a filtering cycle, each filter uses a mixed estimate and measurement to compute a new estimate and a likelihood for each model within the filter. The likelihoods, prior model probabilities, and model switching probabilities are used to compute new model probabilities. The overall state estimate is computed with the new state estimates and their model probabilities. The IMM algorithm for tracking with $N$ models is outlined in the following 5 steps. A derivation and detailed explanation of the IMM algorithm are given in $[2,4]$.

\section{Step 1: Mixing of State Estimates}

The filtering process starts with a prioristate estimates $X_{k-1 \mid k-1}^{j}$, state error covariances $P_{k-1 \mid k-1}^{j}$, and the associated probabilities $\mu_{k-1}^{j}$ for each model. The mixed state estimate for model $j$ at time $k, M_{k}^{j}$, is computed as

$$
X_{k-1 \mid k-1}^{0 j}=\sum_{i=1}^{N} X_{k-1 \mid k-1}^{i} \mu_{k-1 \mid k-1}^{i \mid j}
$$

where

$$
\mu_{k-1 \mid k-1}^{i \mid j}=\frac{1}{\bar{c}_{j}} p_{i j} \mu_{k-1}^{i}
$$

with

$$
\bar{c}_{j}=\sum_{i=1}^{N} p_{i j} \mu_{k-1}^{i}
$$


The mixed error covariance for $M_{k}^{j}$ is computed as

$P_{k-1 \mid k-1}^{0 j}=\sum_{i=1}^{N} \mu_{k-1 \mid k-1}^{i \mid j}\left[P_{k-1 \mid k-1}^{i}+\tilde{X}^{i \mid 0 j}\left(\tilde{X}^{i \mid 0 j}\right)^{T}\right]($

where

$$
\tilde{X}^{i \mid 0 j}=X_{k-1 \mid k-1}^{i}-X_{k-1 \mid k-1}^{0 j}
$$

\section{Step 2: Model Conditioned Updates}

The Kalman or PDA filtering equations provide the model-conditioned updates.

\section{Step 3: Model Likelihood Computations}

The likelihood of $M_{k}^{j}$ for the Kalman filter is given by

$$
\lambda_{k}^{j}=\frac{1}{\sqrt{\left|2 \pi S_{k}^{j}\right|}} \exp \left[-0.5 g_{k}^{j}\right]
$$

and the likelihood for the PDAF is given by

$$
\lambda_{k}^{j}=\left(V_{k}^{j}\right)^{-m_{k}}\left(\left(1-P_{D} P_{G}\right)+\frac{P_{D} P_{G}}{m_{k}}\left(V_{k}^{j}\right) \sum_{i=1}^{m_{k}} \Lambda_{k}^{i \mid j}\right)
$$

Step 4: Model Probabilities Update

The model probabilities are updated as

$$
\mu_{k}^{j}=\frac{1}{c} \lambda_{k}^{j} \bar{c}_{j}
$$

where

$$
c=\sum_{i=1}^{N} \lambda_{k}^{i} \bar{c}_{i}
$$

\section{Step 5: Combination of State Estimates}

The state estimate and error covariance for the IMM algorithm output are obtained from a probabilistic sum of the individual filter outputs and is given by

$$
\begin{aligned}
X_{k \mid k} & =\sum_{i=1}^{N} X_{k \mid k}^{i} \mu_{k}^{i} \\
P_{k \mid k} & =\sum_{i=1}^{N} \mu_{k}^{i}\left[P_{k \mid k}^{i}+\tilde{X}^{i}\left(\tilde{X}^{i}\right)^{T}\right]
\end{aligned}
$$

where

$$
\tilde{X}^{i}=X_{k \mid k}^{i}-X_{k \mid k}
$$

\section{ESA REVISIT TECHNIQUE}

The output of the IMM algorithm is used to calculate the sample time for the next radar measurement. The revisit technique described in this section has been successfully demonstrated for a surveillance application [10]. However, this technique can also be employed for weapons control and other tactical functions. The next required revisit time is determined on-line after each track update as the latest time at which the target can be expected to be in the predicted track gate, which is defined by the radar beamwidth and the length of the range gate in track mode. The revisit time is determined by selecting the minimum of the required revisit times for range, bearing, and elevation. For the angles, the sample periods are computed to be the times at which the standard deviations of the predicted angles exceed a fraction of the beamwidth. The sample period for range is computed to be the time at which the standard deviations of the predicted range exceeds a fraction of the radar range gate. Using the output of the IMM algorithm to compute the next revisit time gives sample periods that automatically reflect target maneuvers, target range, missed detections, radar beam shape, and fluctuating signal amplitudes. A maximum allowable sample period is also computed to ensure that the target is within the beam for the next dwell. When a missed detection occurs the sample period is commanded to be 1 sec to quickly attain a target measurement in order to maintain the track.

The maximum sample period is calculated as the time at which a target maneuvering with a maximum acceleration would exit the predicted region covered by the radar beam and range gate. Consider a target characterized by maximum thrust and turn accelerations, $A_{t h r}$ and $A_{t u r n}$, respectively. Let

$$
\phi=\cos ^{-1}\left(\frac{Y_{k \mid k} \cdot V_{k \mid k}^{T}}{\left\|Y_{k \mid k}\right\|\left\|V_{k \mid k}\right\|}\right)
$$

where

$$
\begin{aligned}
Y_{k \mid k} & =\left[\begin{array}{lll}
x_{k \mid k} & y_{k \mid k} & z_{k \mid k}
\end{array}\right] \\
V_{k \mid k} & =\left[\begin{array}{lll}
\dot{x}_{k \mid k} & \dot{y}_{k \mid k} & \dot{z}_{k \mid k}
\end{array}\right]
\end{aligned}
$$

The potential radial and cross range accelerations, $A_{\text {rad }}$ and $A_{\text {cross }}$, are given by

$$
\begin{aligned}
A_{\text {rad }} & =\max \left\{A_{\text {thr }}|\cos \phi|, A_{\text {turn }}|\sin \phi|\right\} \\
A_{\text {cross }} & =\max \left\{A_{\text {thr }}|\sin \phi|, A_{\text {turn }}|\cos \phi|\right\}
\end{aligned}
$$

The maximum sample period, $T_{s}^{m}$, is given by

$$
T_{s}^{m}=\min \left\{\sqrt{\frac{\left(\theta_{b w}-2 \sigma_{a}\right)}{A_{\text {cross }}}\left\|Y_{k \mid k}\right\|}, \sqrt{\frac{\left(L_{g}-4 \sigma_{s}^{r}\right)}{A_{\text {rad }}}}\right\}
$$

where $\theta_{b u}=\min \left\{\theta_{b}, \theta_{e}\right\}, \sigma_{a}=\min \left\{\sigma_{s}^{b}, \sigma_{s}^{e}\right\}$, and $L_{g}$ is the length of the range gate with $\sigma_{s}^{r}, \sigma_{s}^{b}$, and $\sigma_{s}^{e}$ being the standard deviation of the threshold values for scheduling. 
The sample period is determined on-line as the time when the error covariance of the predicted position exceeds a given threshold. The sample period, $T_{s}$, is computed such that

$$
\bar{P}_{k+1 \mid k} \leq \bar{P}_{t h}
$$

where $\bar{P}_{k+1 \mid k}$ denotes the error covariance of the predicted position and $\bar{P}_{t h}$ the threshold. The term $\bar{P}_{k+1 \mid k}$ is given by

$$
\begin{aligned}
\bar{P}_{k+1 \mid k}= & \sum_{i=1}^{N} \mu_{k}^{i}\left[\bar{F}_{k+1}^{i} P_{k \mid k}^{i}\left(\bar{F}_{k+1}^{i}\right)^{T}+\bar{G}_{k+1}^{i} Q_{k}^{i}\left(\bar{G}_{k+1}^{i}\right)^{T}\right. \\
& \left.+\bar{X}_{k+1 \mid k}^{i}\left(\bar{X}_{k+1 \mid k}^{i}\right)^{T}\right]-\bar{X}_{k+1 \mid k} \bar{X}_{k+1 \mid k}^{T}(6.8)
\end{aligned}
$$

where

$$
\bar{X}_{k+1 \mid k}=\sum_{i=1}^{N} \mu_{k}^{i} \bar{X}_{k+1 \mid k}^{i}
$$

with

$$
\bar{X}_{k+1 \mid k}^{i}=\bar{F}_{k+1}^{i} X_{k \mid k}^{i}
$$

and $N$ is the number of models. The $\bar{F}_{k+1}^{i}$ and $\bar{G}_{k+1}^{i}$ are the state transition and process error matrices for the sample period calculation and contain values that only contribute to position. The term $\bar{P}_{t h}$ is given by

$$
\bar{P}_{t h}=\lambda \bar{R}_{k}^{X Y Z}
$$

with $\lambda$ being a positive scalar and $\bar{R}_{k}^{X Y Z}$ is approximated by

$$
\begin{aligned}
\bar{R}_{k}^{X Y Z} & =U_{k}\left[\begin{array}{ccc}
\left(\sigma_{s}^{r}\right)^{2} & 0 & 0 \\
0 & \left(\sigma_{s}^{b}\right)^{2} & 0 \\
0 & 0 & \left(\sigma_{s}^{e}\right)^{2}
\end{array}\right] U_{k}^{T} \\
& =U_{k} \bar{R}_{k}^{R B E} U_{k}^{T}
\end{aligned}
$$

where $U_{k}$ is a rotation matrix computed with the values of $X_{k \mid k}$. The threshold and predicted error covariance of position in spherical coordinates, $\bar{P}_{t h}^{S p}$ and $\bar{P}_{k+1 \mid k}^{S p}$, respectively, are given by

$$
\begin{aligned}
\bar{P}_{t h}^{S p} & =U_{k}^{T} \bar{P}_{t h} U_{k}=\lambda \bar{R}_{k}^{R B E} \\
\bar{P}_{k+1 \mid k}^{S p} & =U_{k}^{T} \bar{P}_{k+1 \mid k} U_{k}
\end{aligned}
$$

The sample period for each coordinate, $T_{s}^{i}$, is calculated using the diagonal elements of $\bar{P}_{t h}^{S p}$ and $\bar{P}_{k+1 \mid k}^{S p}$, since those elements correspond to range, bearing, and elevation, and explicitly solving for $T_{s}^{i}$. The output sample period, $T_{s}$, is $T_{s}=\min \left\{T_{s}^{r}, T_{s}^{b}, T_{s}^{e}\right\}$. The output sample time, $T_{k+1}$, is given by $T_{k+1}=T_{k}+T_{s}$. The sample period calculation is performed after each update of the ESA track and the time for the next radar dwell is scheduled accordingly. When additional sensors are supporting the track, a new revisit interval is computed after each track update and the ESA is commanded to delay its initial update a specified amount until the ESA is required to perform a dwell.

\section{Simulation RESUltS}

Simulation results that demonstrate resource management of an ESA through multisensor integration are presented in this section. The focus of the study is to demonstrate that the radar resources required to track maneuvering targets can be reduced when additional sensors support the ESA track for a surveillance application. The sensor suite for the simulation includes an ESA and a sensor providing angle measurements, such as an IRST or a PESM. The search detections generated by the ESA that correlate with the track are also integrated. As in [10], the performance criteria is to minimize the time-energy budget of the ESA. This is accomplished by using a minimum number of dwells at the lowest possible energy. A comparison with a conventional approach is not presented in this paper because the IMM algorithm provides more accurate state estimates [5].

The benchmark problem for radar resource allocation [9] forms the algorithm testbed. The benchmark problem is comprised of tracking requirements, an ESA model to generate measurements, and a tracking algorithm. The tracking requirements are specified in terms of limits on target position and maneuverability. The tracking algorithm is designed by the researcher and must adapt to the tracking environment. Proper beam-pointing control, which consists of pointing the radar beam and selecting the waveform energy, is required to receive measurements from the target. The radar model of [9] was upgraded to include dwells with multiple distinct frequencies. The use of multiple frequencies provides a more stable Signal-to-Noise Ratio (SNR) by reducing the effect of the radar cross section fluctuations. Performance characteristics of the ESA model are presented in Fig. 7.1 and include the one-way and two-way beamwidths, and typical measurement standard deviations for perfect pointing. The benchmark testbed was also modified to include measurements from additional sensors. Since sensor models with the complexity of the benchmark problem were not available, the measurements for the PESM or IRST are created by corrupting the absolute measurement with white Gaussian errors of a given standard deviation. To simplify the simulation, all the sensors are assumed to be located on the same platform and only one additional sensor is allowed to provide its information to the ESA.

The flight profile for the target is presented in Fig. 7.2 and is representative of a medium bomber flying at high speed with good maneuverability. The target maintains 

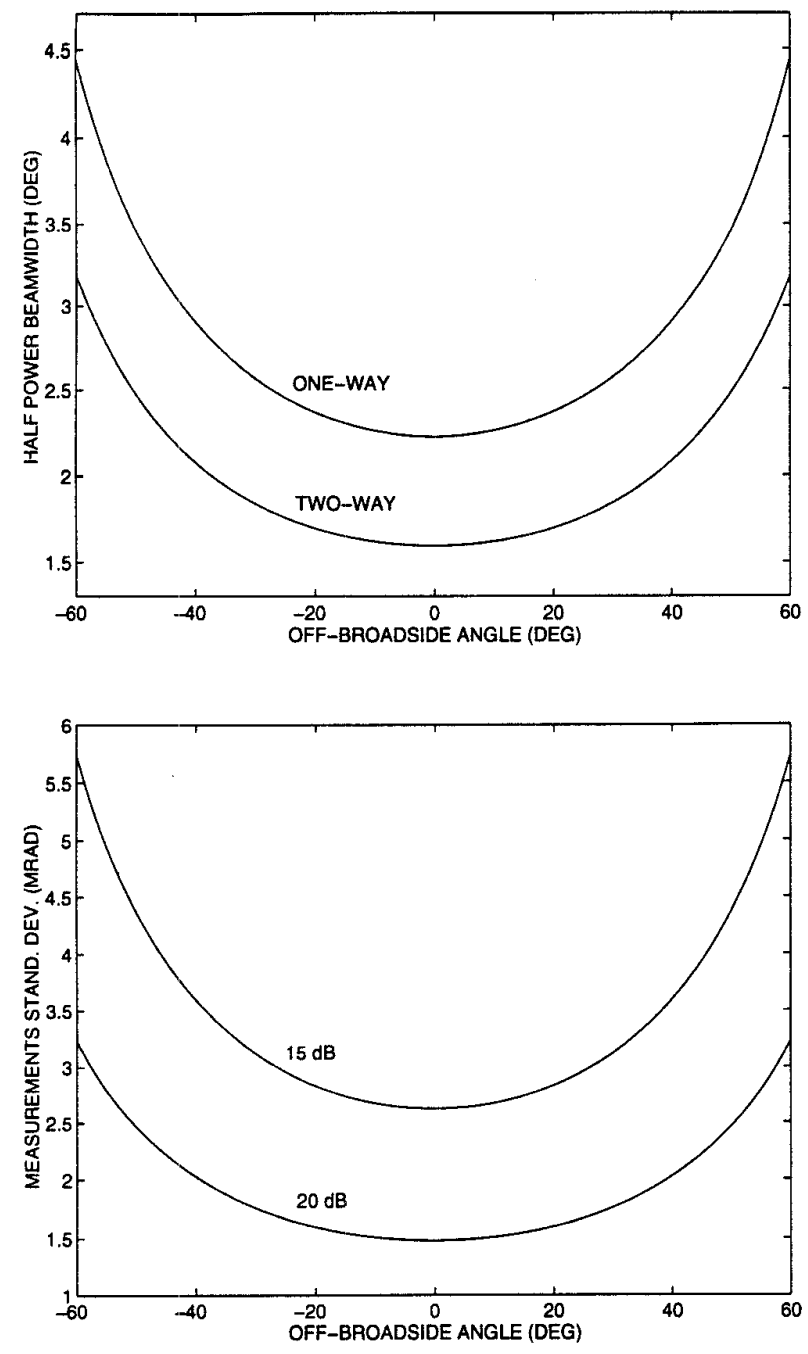

Figure 7.1 ESA Performance Characteristics

a speed of $251 \mathrm{~m} / \mathrm{s}$ and an altitude of $2.29 \mathrm{~km}$ for the first $30 \mathrm{~s}$. A $4 \mathrm{~g}$ turn is performed through a course change of 45 degrees. After another $30 \mathrm{~s}$, a $6 \mathrm{~g}$ turn is performed as the throttle is increased to full afterburner. The aircraft pitches up and climbs to an altitude of $4.57 \mathrm{~km}$. Following the climb, straight and level, nonaccelerating flight is maintained for the completion of the trajectory.

The beam-pointing control for the ESA is provided by an IMM algorithm with kinematic models that consist of two constant velocity models and a constant turn rate model and is designed similar to the estimator presented in [10]. When adaptive sampling is requested, the output of the IMM algorithm is used to determine the appropriate revisit time for the next radar dwell. The position to point the radar is calculated with Eqs. (6.9) and (6.10) by inserting the computed sample period. A median estimator for a sliding window of target returns is used to select the appropriate waveform for an ESA dwell to
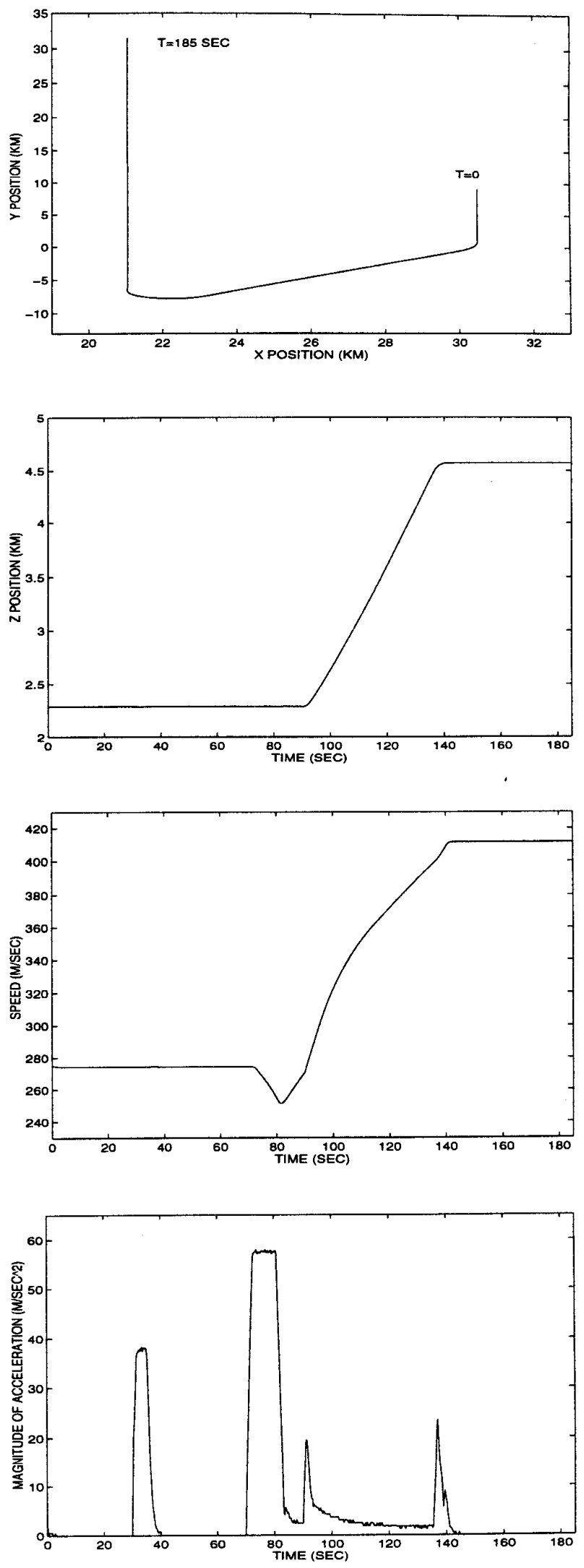

Figure 7.2 Trajectory Profile 
meet a given operating condition. The sliding window consists of the latest four SNR values obtained from the sets of ESA measurements employed to update the track.

A performance comparison is presented for a conventional estimator and a IMM estimator for a constant sample period of $1.0 \mathrm{~s}$, since a conventional estimator typically requires this rate when tracking targets of this type. The Kalman filter is used by both the conventional an IMM estimators in the following examples since the estimation error similar when the PDAF is employed. The performance criteria for this section is the accuracy of the state estimates. The conventional estimator is a Kalman filter employing a Constant Velocity (CV) kinematic model. The process error covariance for the $\mathrm{CV}$ estimator is $Q_{k}=900 \mathrm{I}_{3} \mathrm{~m}^{2} / \mathrm{s}^{4}$. The IMM estimator is designed like the one in [10] and has process error covariances of $Q_{k}^{1}=4 I_{3} \mathrm{~m}^{2} / \mathrm{s}^{4}, Q_{k}^{2}=900 I_{3} \mathrm{~m}^{2} / \mathrm{s}^{4}$, and $Q_{k}^{3}=38 I_{3} \mathrm{~m}^{2} / \mathrm{s}^{6}$, where 1 refers to $C V 1,2$ refers to CV2, and 3 refers to the turning rate model (TR). The TR acceleration estimate is modified if its turning rate is not greater than $0.03 \mathrm{rad} / \mathrm{s}$ for filtering and prediction. The initial model probabilities are

$$
\mu_{0}=\left[\begin{array}{lll}
0.8 & 0.1 & 0.1
\end{array}\right]
$$

with model switching probabilities

$$
\mathrm{II}=\left[\begin{array}{ccc}
0.92 & 0.07 & 0.01 \\
0.1 & 0.75 & 0.15 \\
0.05 & 0.03 & 0.92
\end{array}\right]
$$

The Root-Mean-Square-Errors (RMSEs) of the two estimators for 100 Monte Carlo experiments are given in Fig. 7.3a. The IMM estimator provides better track performance by having less estimation error as presented in Fig. 7.3a. The process error for the CV estimator could have been chosen in order to match the performance of the IMM estimator in the nonmaneuvering regions, but its estimation error during maneuvers would be larger than the IMM estimator. The IMM estimator also provides mode estimation, as shown in Fig. 7.3b. Notice that the CV model with small process error, CV1, is dominant during nonmaneuvering portions of the flight. The maneuver response model, $\mathrm{CV}$ with high process error, CV2, and the TR model respond during maneuvers very effectively. The filter generated standard deviations for each estimator are given in Fig. 7.3c and are the square root of the sum of the position or velocity elements from the diagonal of the error covariance matrix. Unlike the CV estimator, the standard deviations for the IMM estimator change to reflect that the target is maneuvering. The standard deviations for the IMM estimator are smaller than those of the CV estimator in the nonmaneuvering region and increase in the maneuvering regions to reflect a greater uncertainty in the state estimates. The cumulative radar time is also presented in Fig 7.3d. Since an operating SNR of $18 \mathrm{~dB}$ was selected for all experiments, the behavior exhibited for radar time and radar energy are very similar. The amount of radar time for both estimators is very similar since the revisit interval is the same.

A sensor providing angle measurements (i.e., bearing and elevation) is integrated with the ESA and a performance comparison is presented for the conventional and IMM estimators. The sample period of the ESA and angle sensor is $1.0 \mathrm{~s}$. The accuracy of the angle measurements is $0.5 \mathrm{mrad}$. An IRST typically has more accurate measurements and a PESM provides measurements that are usually less accurate than the $0.5 \mathrm{mrad}$ and the results can be adjusted depending on the accuracy of the angle measurements. The RMSEs are given in Fig. 7.4a and are compared to IMM estimator with the ESA only. The IMM estimator provides better track performance by having less estimation error as presented in Fig. 7.4a. The conventional estimator employing multiple sensors has an error that is similar to the IMM estimator employing only ESA measurements for most of the trajectory. Unlike the conventional estimator, the velocity error of the IMM estimator does not significantly improve with the inclusion of the additional sensor. The IMM estimator also provides mode estimation, as shown in Fig. 7.4b. Notice that the model probabilities are different when compared to Fig. 7.4b. The filter generated standard deviations for each estimator are given in Fig. 7.4c. The filter generated standard deviations from the multiple sensors are smaller than the single sensor case and is reflected in a smaller estimation error. The standard deviations for the IMM estimator are smaller than those of the CV estimator in the nonmaneuvering region and increase in the maneuvering regions to reflect a greater uncertainty in the state estimates. The oscillations in the standard deviations are due to the sensors providing measurements with different accuracies. Notice that the oscillations of the IMM estimator are less than the conventional estimator. The cumulative radar time is also presented in Fig. 7.4d and is the same as the single sensor case since the revisit time of the radar did not change.

The sample period for the IMM estimator with adaptive sampling is given in Fig. 7.5a. Since the objective of adaptive sampling for surveillance is to maintain track of the target, the estimation error is not presented since it is be significantly larger when compared to a periodic revisit technique. The sample period and cumulative radar energy are presented since these measures of performance characterize the requirements of the track filter in requesting information (i.e., measurements) from the ESA. The results are an average of 250 Monte Carlo experiments. The conventional estimator, when initiation is considered, revisits the target on average 185 times per run. If no constraint is placed upon the target 

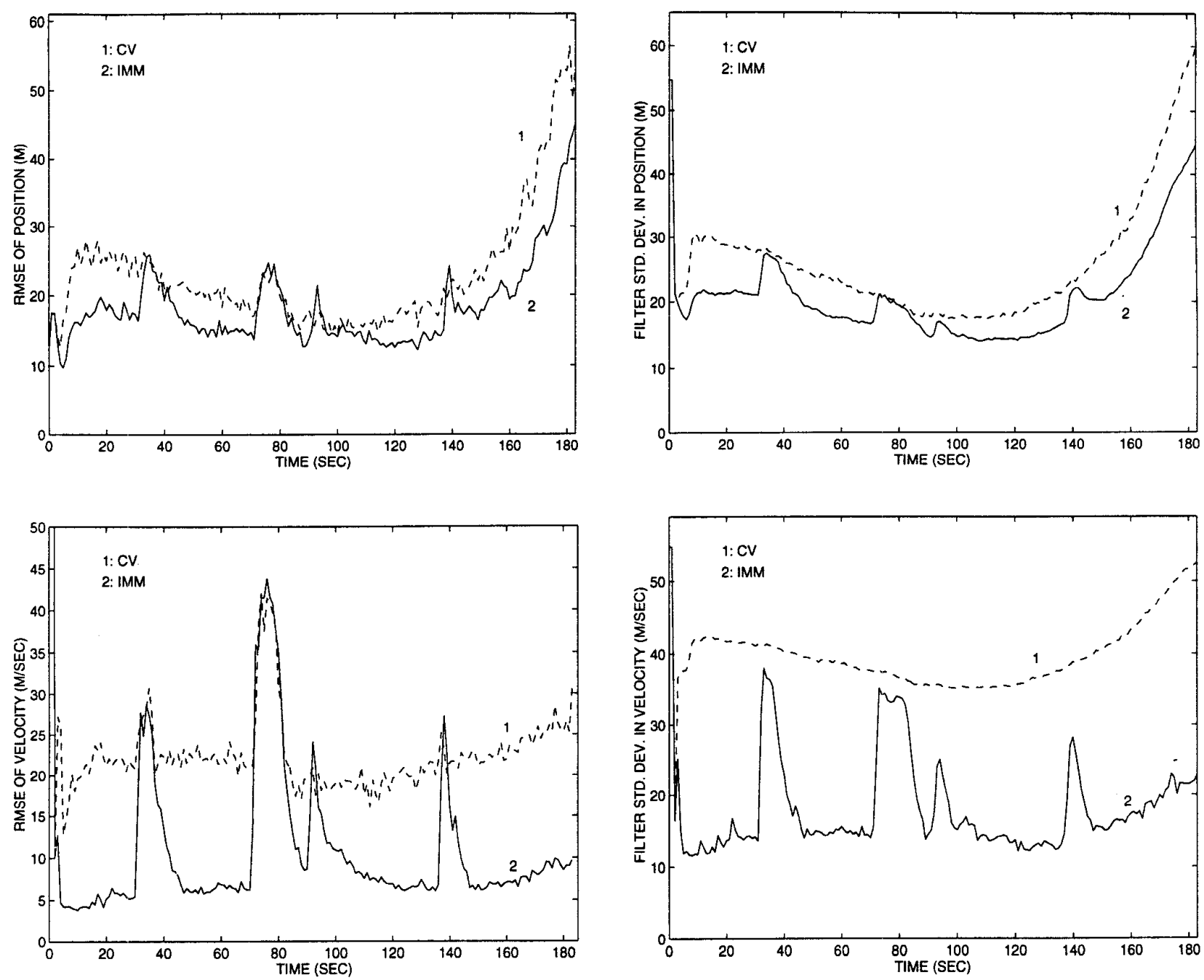

Figure 7.3a Estimation Error

Figure 7.3c Filter Standard Deviation

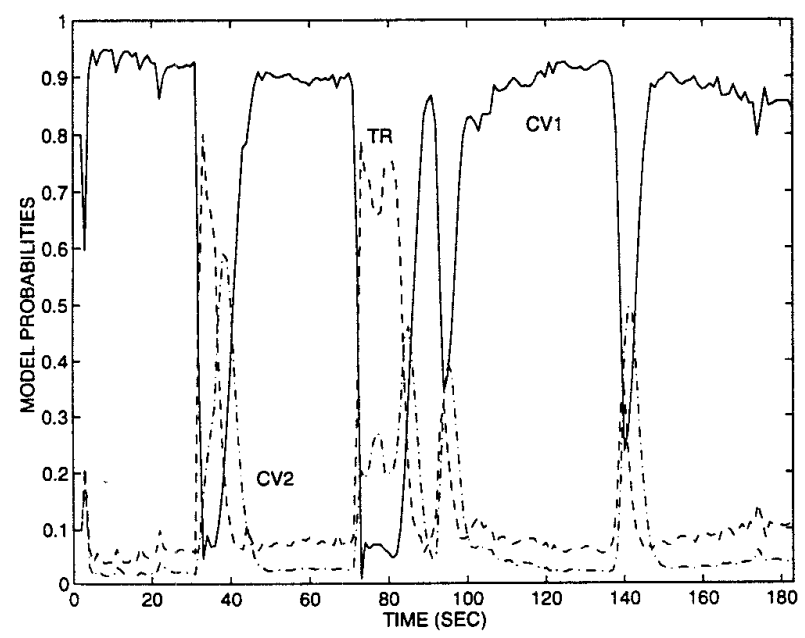

Figure 7.3b Model Probabilities

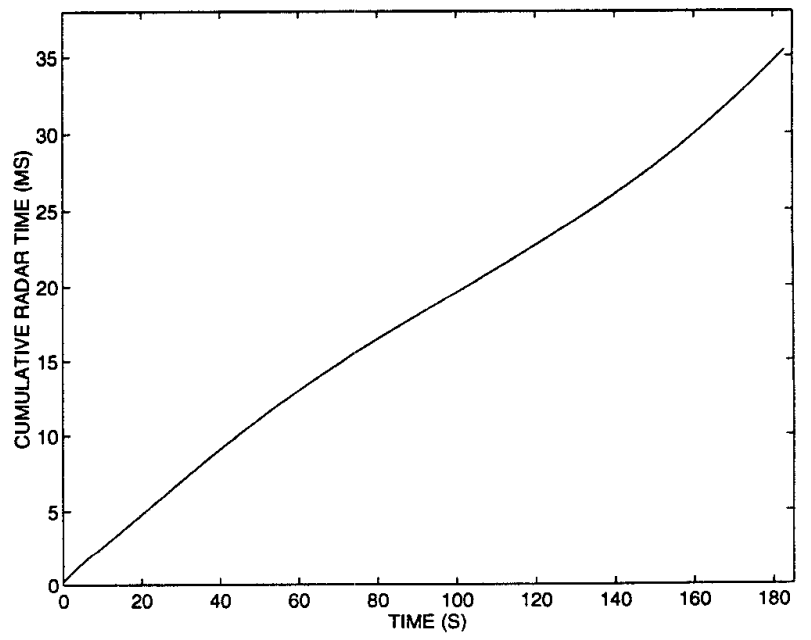

Figure 7.3d Cumulative Radar Time 

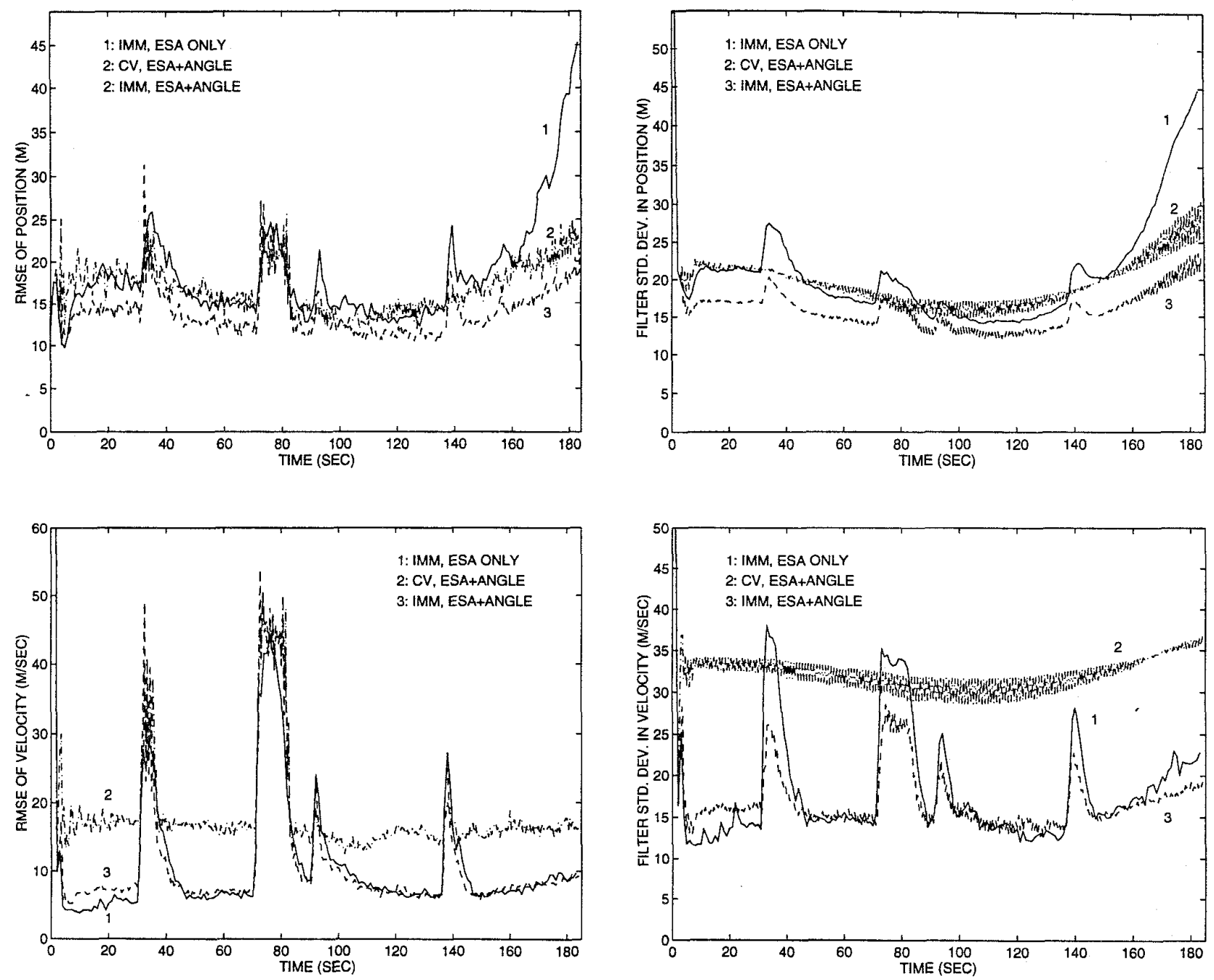

Figure 7.4a Multisensor Estimation Error

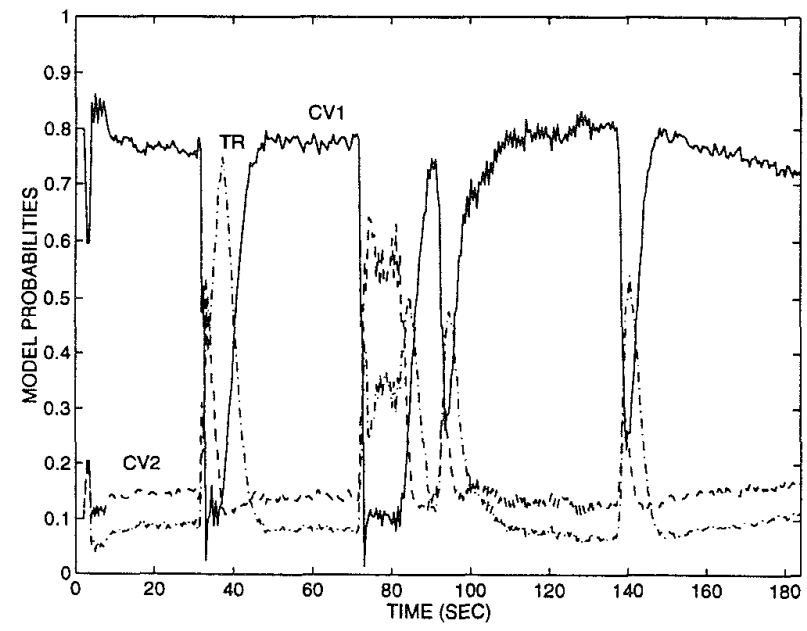

Figure 7.4b Multisensor Model Probabilities

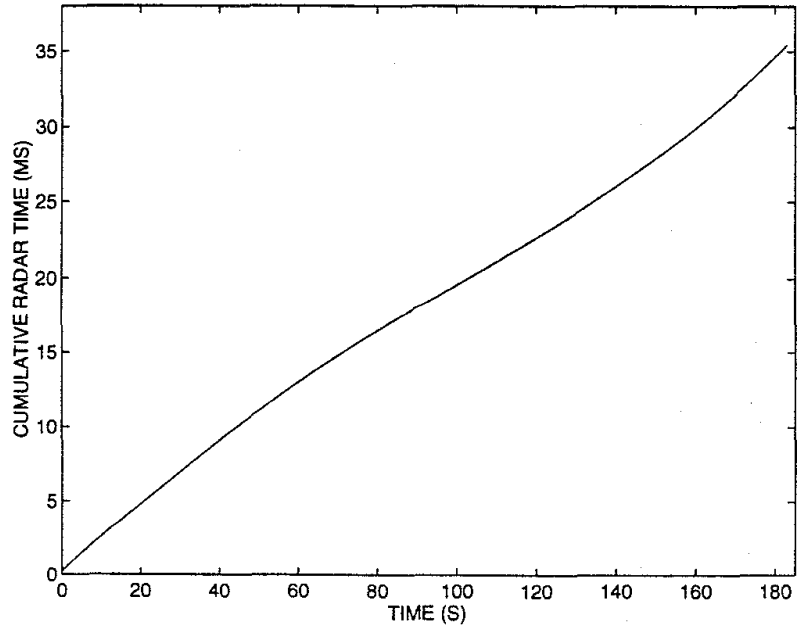

Figure 7.4d Multisensor Cumulative Radar Time 
revisit interval, the IMM algorithm with adaptive sampling revisits the target an average of 58 times per run, which is a savings of $69 \%$ when compared to a conventional revisit technique. The sample period is significantly smaller when the target is maneuvering, however it is not less than the sample period required by the conventional technique. The lines are not smooth since because a different sequence of measurements is selected for each Monte Carlo experiment. The scheduling parameter $\lambda$ is 1.0 for these results. The maneuverability of the target and its ability to escape from the radar beam is not considered. The target may be lost because the sample periods can be very large when the onset of a maneuver occurs and measurements may not be able to be obtained in order to maintain track. The maneuverability of the target is incorporated through the maximum allowable sample period calculation. When the maneuverability of the target is considered, an average of 67 revisits per run are required, which is a savings of $64 \%$ when compared to the conventional technique. Notice that the sample period during maneuvers is the same while the shape of the beam has limited the sample period when the target is not maneuvering. The maneuverability for this set of results is $A_{t h r}=20 \mathrm{~m} / \mathrm{s}^{2}$ and $A_{t u r n}=70 \mathrm{~m} / \mathrm{s}^{2}$. The cumulative radar time is presented in Fig. 7.5b. The use of adaptive sampling has significantly reduced the amount of radar time required to track the target. The maximum sample period is a system constraint that is required in order to maintain track and thus needs to be employed for subsequent examples.

The sensitivity to the selection of the maximum sample period parameters is presented next. The effect upon the sample period due to the length of the range gate is presented in Fig. 7.6a. Notice that the maximum sample period increases as the length of the range gate increases from $1.0 \mathrm{~km}$ to $2.0 \mathrm{~km}$. Notice that there is no effect upon the sample period during maneuvers. The effect on the sample period due to the maximum target maneuverability is presented in Fig. 7.6b. Notice that the maximum sample period increases as the maneuverability of the target decreases, which is indicated by the thrusting and turning accelerations. Notice that there is again no effect upon the sample period during maneuvers. The selection of the parameters is critical to attaining the largest possible revisit interval.

The effect upon the radar-time energy by incorporating the PDAF into the adaptive sampling process is presented next. The NN PDAF is employed for this study. It is expected that the sample would be smaller with the inclusion of the PDAF since the probability concerning the origin of the measurement, $\beta$, is less than one. The effect upon the sample period due to the PDAF is presented in Fig. 7.7a. Notice that the sample period is effected only during target maneuvers since there is

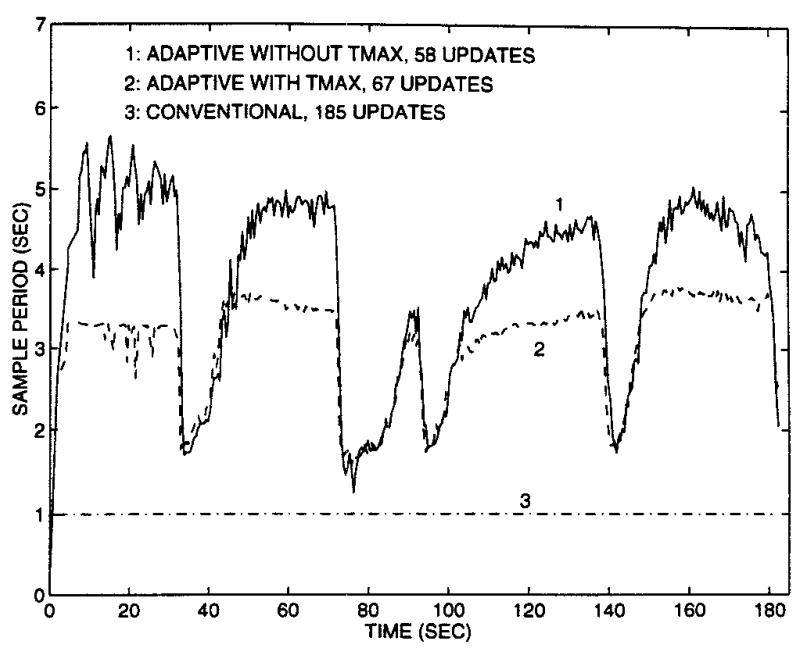

Figure 7.5a Adaptive Revisit Intervals

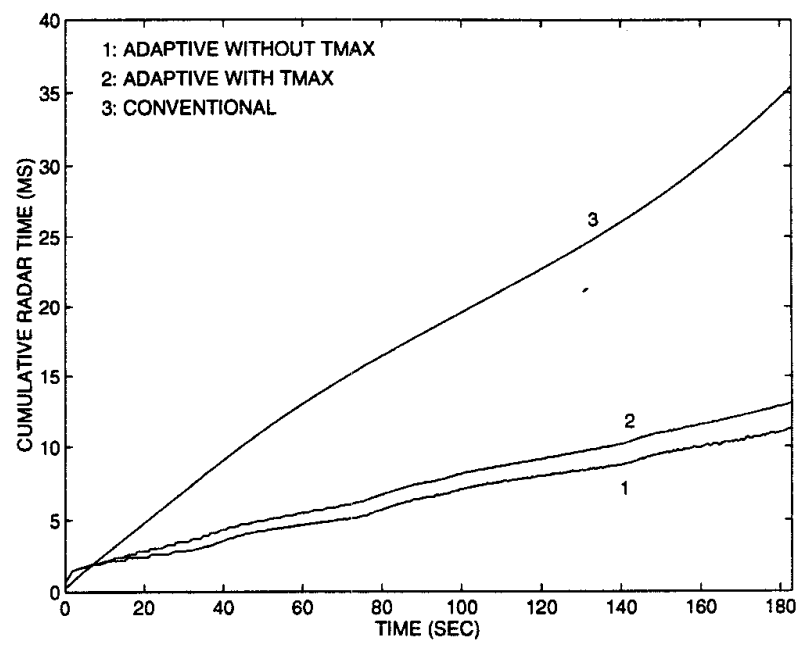

Figure 7.5b Adaptive Cumulative Radar Time

a greater uncertainty associated with the measurement. The cumulative radar time, presented in Fig. $7.7 \mathrm{~b}$, deviates when the maneuver occurs but otherwise requires the same amount of radar time as the Kalman filter. However, the PDAF will lose fewer tracks when clutter is introduced to the simulation since is does not make the "hard" decision associated with the Kalman filter.

An initial reduction in the time-energy required to track the targets observed by the ESA can be realized by employing the detections provided by the search function. This reduction can occur since the search function inevitably obtains measurements from the targets that are already in track. The number of track dwells saved in a given period of time is dependent on the search frame rate and there is no increased usage of the radar because search dwells occur as a matter of course. An example that exhibits the use of search detections to conserve 


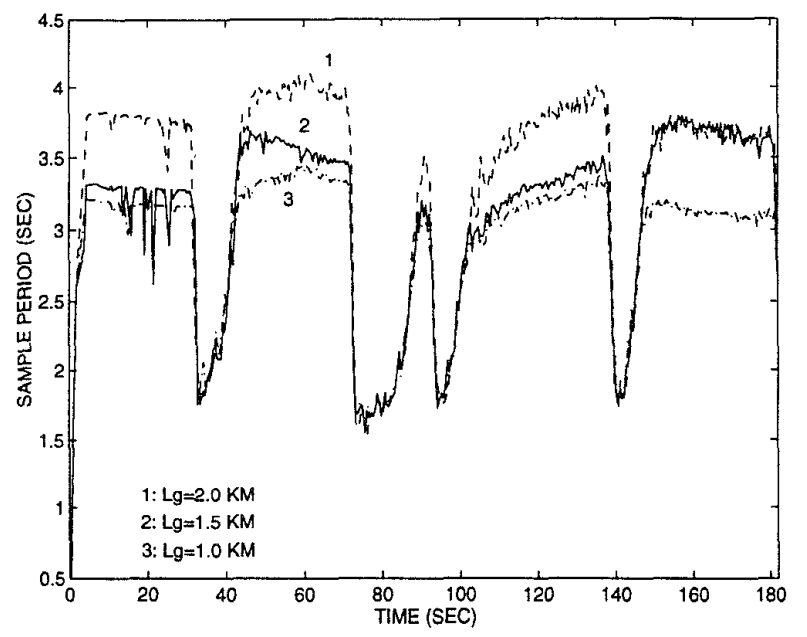

Figure 7.6a Effect of Range Gate Length

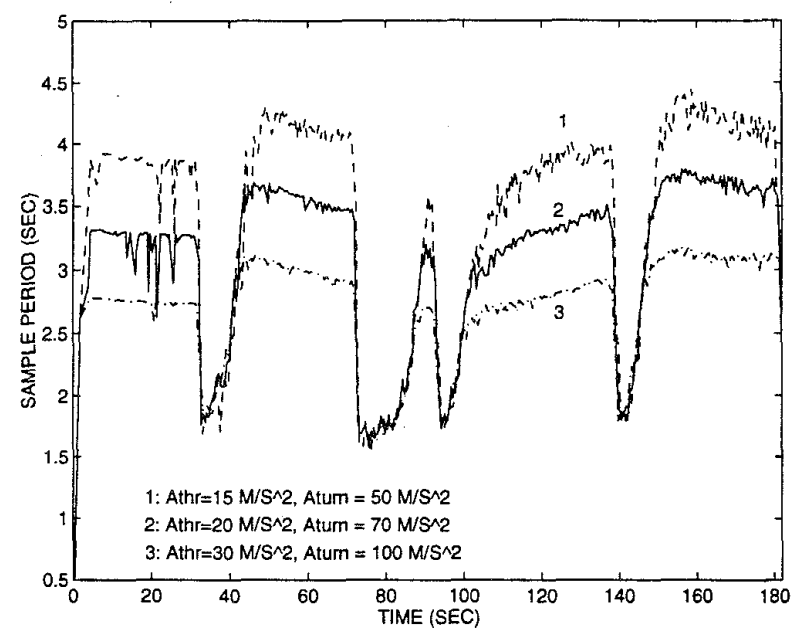

Figure 7.6b Effect of Target Maneuverability

track function resources is presented in Fig. 7.7a. For the simulation, the search detections are provided at $10 \mathrm{~s}$ intervals. The reduction in the time-energy required to track by including range-only updates from the search function is not significant. However, if the measurement includes monopulse processing and the range, bearing, and elevation are used to update the target track, a significant increase in the required time between track updates occurs. The reduction of the radar time-energy for including monopulse processed search detections is also presented in Figs. 7.7a and 7.7b. There is a $12 \%$ reduction in updates when the monopulse processed search detections are used.

The next set of results demonstrate the potential savings in the radar time-energy when an external sensor is integrated with the ESA. The IRST or PESM and the ESA are tracking common targets and the tracks are

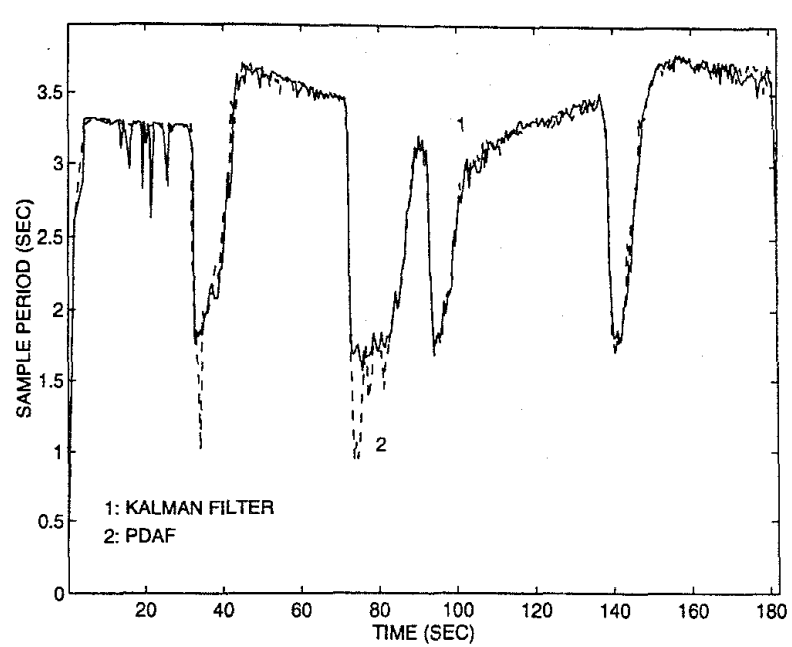

Figure 7.7a Revisit Interval with PDAF

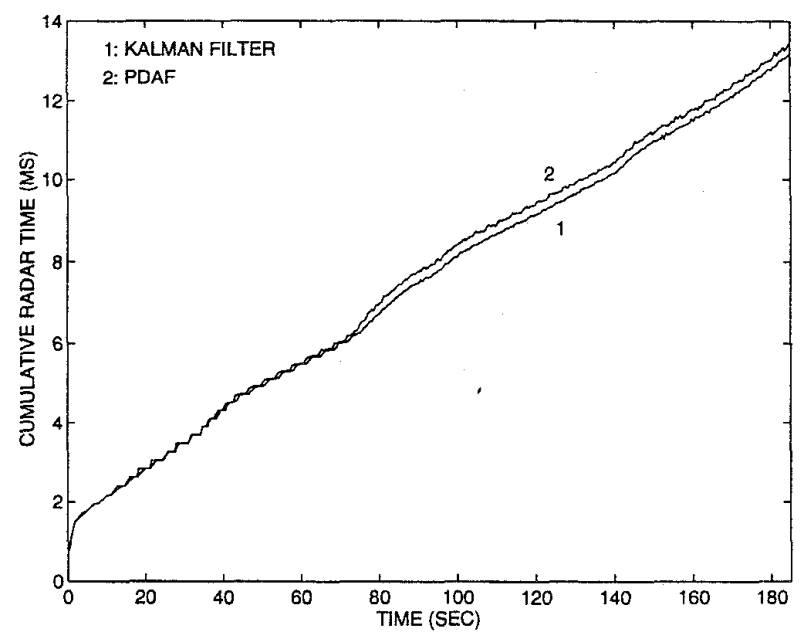

Figure 7.7b Radar Time with PDAF

assumed correlated. These angle sensors provide bearing and elevation measurements and a partial update (range is not updated and therefore its uncertainty grows) of the existing track is performed. Since a partial update is accomplished, the time for the next track dwell can be delayed. However, the range measurement from the search dwell will be used to update the track and reduce the uncertainty in range. A track dwell is employed only when necessary under this configuration.

The angle measurement are provided at $1 \mathrm{~s}$ intervals with uncertainties of approximately $0.5 \mathrm{mrad}$. As presented in Figs. 8.8a and 8.8b, a large reduction in the radar time-energy is accomplished by integrating the angle sensor with the ESA. The ESA is performing normal track processing (i.e., no search detections) and the angle measurements are integrated into the estimation process. A time savings of $55 \%$ is accomplished when com- 


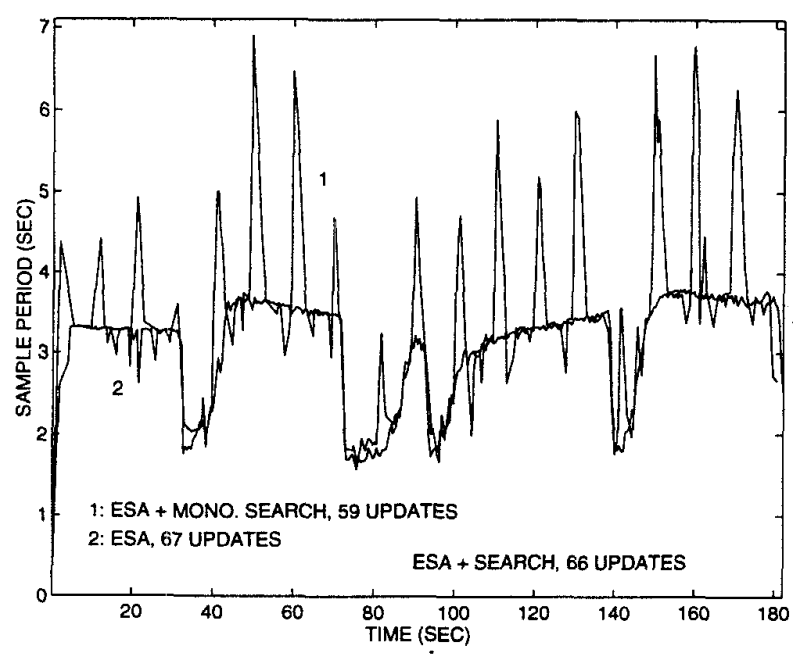

Figure 7.7a Revisit Interval with Search

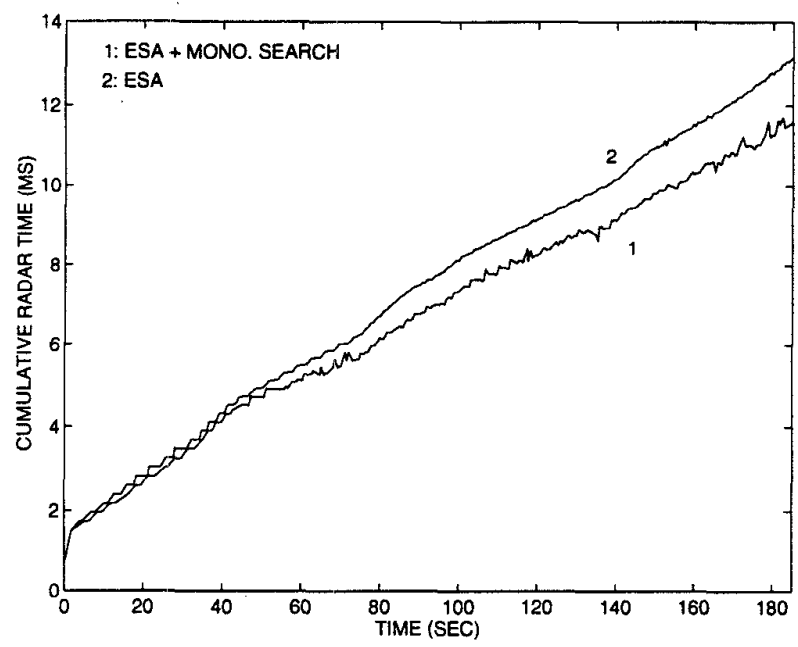

Figure 7.7b Radar Time with Search

pared to the radar-only IMM estimator employing adaptive sampling. Additional time savings occurs when the range measurements from the search function are used. A $60 \%$ savings is accomplished under these conditions and are presented in Fig. 6.4. Notice that the cumulative radar time is greatly reduced when the angle sensor can support the ESA. Notice that the sample period is large when the target is not maneuvering and similar to the radar-only performance when the target is maneuvering.

The search detections of the ESA are integrated with the angle sensor to further reduce the ESA resource requirement for tracking and the results are presented in Figs. 7.9a and 7.9b. The largest conservation of ESA resources occurs when the range measurements from the search function and the angle measurements are used in conjunction with the track function data. An $75 \%$ savings is accomplished when the range measurements

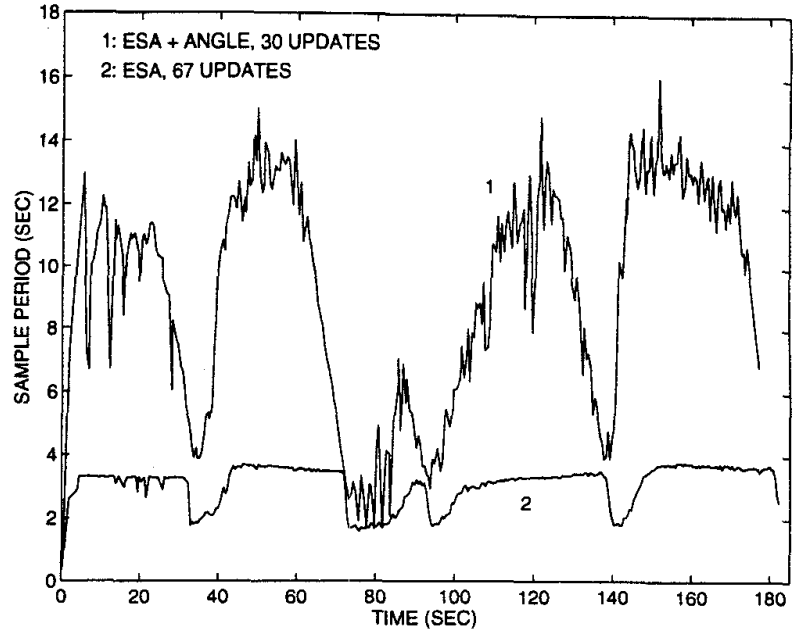

Figure 7.8a Reviṣit Interval with Angle Sensor

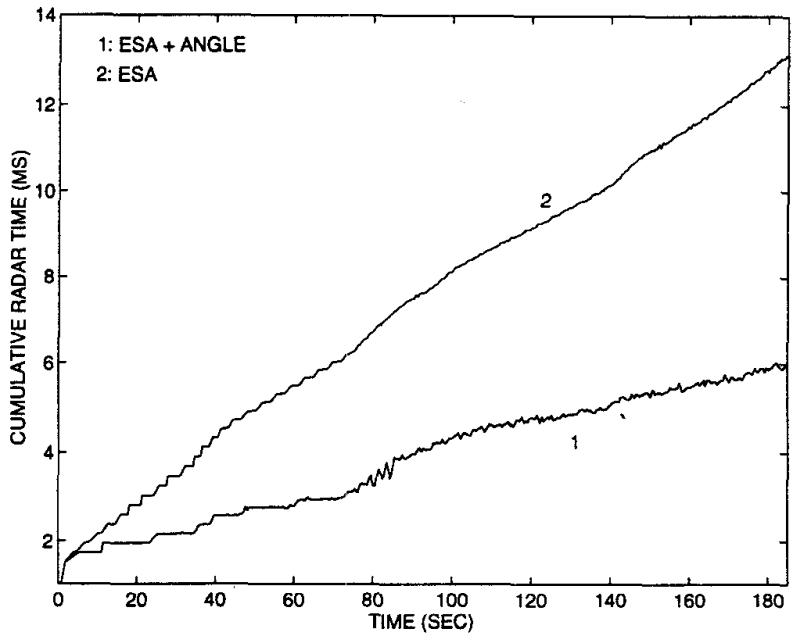

Figure 7.8b Radar Time with Angle Sensor

from search function are also considered, as presented in Fig. 7.9a. The accurate angle measurements and the high data rate are the largest contributing factors for reducing time requirements of the ESA.

\section{CONCLUDING REMARKS}

A technique for tracking maneuvering targets and multisensor integration for ESA resource management has been presented. The proposed technique avoids the decision-directed logic associated with conventional techniques by employing the IMM algorithm. The resources for the ESA required to track maneuvering targets can be reduced by allowing the system function to request the amount of information needed for completion of its task. This can be accomplished with the adaptive revisit technique presented in this paper. If the ESA and other sensors are tracking common targets, the ESA resources 


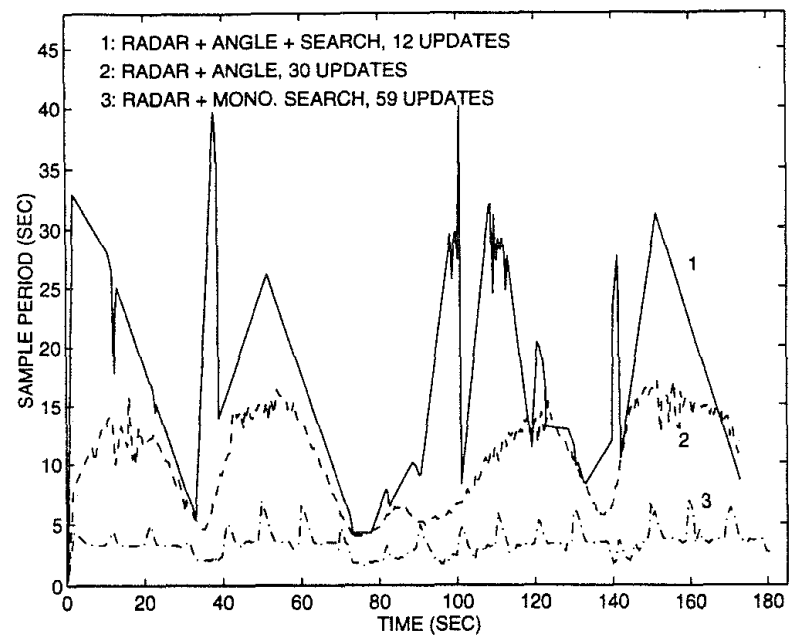

Figure 7.9a Revisit Interval with Multiple Sensors

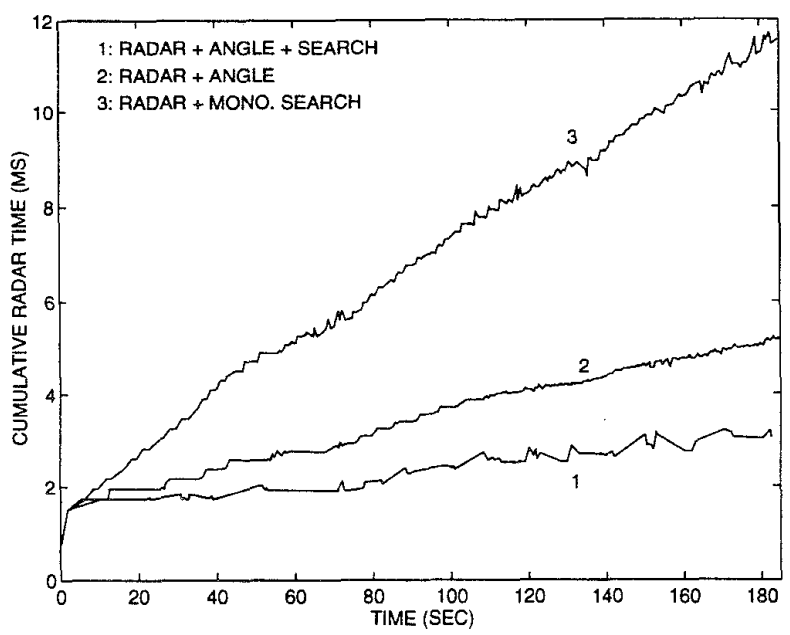

Figure 7.9b Radar Time with Multiple Sensors

needed to track those targets can be reduced through sensor integration by exploiting the potential synergism between the sensors. The revisit technique employed for a single sensor is readily adapted to the multisensor environment. The simulation results demonstrate that through proper sensor integration the radar resources required to track maneuvering targets can be significantly reduced. By continually determining a new revisit time after each track update, the ESA has already scheduled the next required dwell even if the supporting sensors fail to report. By employing this method, fewer tracks will be lost and the state estimates will be more accurate. The technique has been applied to a surveillance application but can be used for other tactical functions, such as weapons control which requires a minimum tracking error, and possibly Theater Ballistic Missile Defense (TBMD). The selection of the tracking parameters for adaptive sampling was explored to illustrate their effects on the sample period and the radar time-energy budget. The inclusion of the PDAF for adaptive sampling was also presented. The PDAF demonstrated similar performance as the Kalman filter when the target is not maneuvering but requires more updates when the target maneuvers. However, the PDAF will lose fewer tracks in a false alarm and clutter environment. Further radar savings could be realized if the inherent search detections of the ESA are provided at a faster rate. Additional effort is needed to acquire IRST and PESM models that can be integrated into the benchmark testbed. Further research includes applying this technique to other tactical functions, incorporating multiple platforms, introducing sensor and location biases, multipath, and tracking multiple, unresolved targets.

\section{REFERENCES}

1. W.D. Blair and Y. Bar-Shalom, "Tracking Maneuvering Targets with Multiple Sensors: Does More Data mean Better Estimates?," IEEE Transactions on Aerospace and Electronic Systems, January 1996.

2. H.A.P. Blom, "An Efficient Filter for Abruptly Changing Systems," Proceedings of $23^{\text {rd }}$ IEEE Conference on Decision and Control, Las Vegas, NV, December 1984.

3. Y. Bar-Shalom, C.Y. Chang, and H.A.P. Blom, "Tracking a Maneuvering Target Using Input Estimation Versus The Interacting Multiple Model Algorithm," IEEE Transactions on Aerospace and Electronic Systems, AES-25, 1989.

4. Y. Bar-Shalom and X.R. Li, Estimation and Tracking: Principles, Techniques, and Software, Artech House, Inc., Boston, MA, 1993.

5. G.A. Watson and W.D. Blair, "Tracking Maneuvering Targets with Multiple Sensors Using the Interacting Multiple Model Algorithm," Proceedings of SPIE Signal and Data Processing of Small Targets 1993, Orlando, FL, April 1993.

6. T. Kirubarajan, Y. Bar-Shalom, and E. Daeipour, "Active Beam Pointing Control of a Phased Array Radar in the Presence of ECM and False Alarms Using IMMPDAF," Proceedings of 1995 American Control Conference, Seattle, WA, June 1995.

7. G.A. Watson and W.D. Blair, "IMM Algorithm for Solution to Benchmark Problem for Tracking Maneuvering Targets," Proceedings of SPIE Signal and Data Processing of Small Targets 1994, Orlando, FL, April 1994.

8. S.S. Blackman, M.T. Busch, and R.F. Popoli, "IMM/MHT Tracking and Data Association for Benchmark Tracking Problem," Proceedings of 1995 American Control Conference, Seattle, WA, June 1995.

9. W.D. Blair and G.A. Watson, "Benchmark Problem for Radar Resource Allocation and Tracking Maneuvering Targets in the Presence of ECM," NSWCDD/TR-96/10, September 1996. 
10. W.D. Blair, G.A. Watson, et. al., "InformationBased Radar Resource Allocation: FY96 Test-OfConcept Experiment (TOCE)," Naval Surface Warfare Division Dahlgren Division, NSWCDD/TR97/22, February 1997.

Gregory A. Watson received a $B S$ in Physics from West Chester University in 1988 and MS in Physics from the University of Connecticut in 1990. In 1990, Mr. Watson joined the Naval Surface Warfare Center Dahlgren Division, Dahlgren, Virginia as a physicist. His work involves target trajectory estimation using multiple model techniques, multisensor integration, target trajectory prediction and generation, resource allocation in a multisensor-multitarget environment, and radar signal processing. 